Sri Lankan Journal of Anaesthesiology 16(2) : 85 - 88 (2008)

\title{
SURVIVING SEVERE SEPSIS - EARLY RECOGNITION AND TREATMENT
}

\author{
Fazal Hameed Khan* \\ Professor, Department of Anaesthesia, Akuh, Karachi ,Pakistan \\ *Corresponding author: E-mail fazal.hkhan@aku.edu
}

Key words: severe sepsis early recognition treatment

\section{INTRODUCTION}

Severe sepsis is defined as sepsis associated with organ dysfunction or organ hypoperfusion and is a major cause of morbidity and mortality throughout the world. In United States more than 500 patients die from severe sepsis each day ${ }^{1}$. This incidence can be very high in less developed countries because of the lack of diagnostic facilities and treatment resources.

In order to simplify the definitions of terms associated with sepsis, the Society of Critical Care Medicine and American College of Chest Physicians proposed consensus definitions in 1992 and these are currently accepted world over $^{2}$.

The Consensus Conference defined sepsis as the presence or presumed presence of an infection accompanied by systemic inflammatory response syndrome (SIRS). SIRS was defined as the presence of two or more of the following:

1. Temperature above $38^{\circ} \mathrm{C}$ or below $36^{\circ} \mathrm{C}$

2. Heart rate above 90 beats/min

3. Respiratory rate above 20 breaths/min (or $\mathrm{PaCO}_{2}<32 \mathrm{mmHg}$, when ventilated) and

4. WCC count greater than $12000 / \mathrm{mm}^{3}$ or less than $4000 / \mathrm{mm}^{3}$ or greater than $10 \%$ immature band forms

The consensus conference definition of severe sepsis is the presence of sepsis based on SIRS criteria and one or more sepsis related organ dysfunction. Septic shock has been defined as the presence of sepsis along with hypotension despite adequate fluid resuscitation.

EARLY RECOGNITION: The initial presentation of severe sepsis is often non specific. Patients with relatively benign or mild infection can deteriorate very rapidly needing intensive care. The transition from sepsis to septic shock occurs during the first 24 hours after admission. This transition results in increase morbidity and mortality ${ }^{4}$.

It is important to recognize patients at risk for developing severe sepsis early. When these patients are recognized early, the sepsis bundles can be initiated and this can result in decreasing the morbidity or mortality related to severe sepsis.

The clinicians should have a high index of suspicion in any patient who is admitted with suspected infections and 2 or more SIRS criteria. All patients with a suspected infection should have vital signs and a white blood cell and differential count, measured as soon as possible. A systematic review is done to reveal sepsis induced organ dysfunction. These organ dysfunction can be identified as evidence of acute lung injury manifested by a $\mathrm{PaO}_{2} / \mathrm{FiO}_{2}$ ratio of less than 300, coagulation abnormalities, thrombocytopenia, altered mental status, renal, liver or cardiac failure, hypoperfusion with lactic acidosis and hypotension unresponsive to fluid resuscitation.

\section{MANAGEMENT GUIDELINES FOR SEVERE SEPSIS}

As a result of the collaborative approach between the society of Critical Care Medicine, the ESICM, and the International Sepsis Forum, the surviving sepsis campaign was formed in 2002 with the aim of decreasing the morality due to sepsis by $25 \%$. The surviving sepsis campaign took initiative to convince physician for early recognition and treatment of severe sepsis.

In second phase of the surviving sepsis campaign an international consensus committee from 
several international organizations was created and evidence based guidelines were elaborated. These are known as "Sepsis Bundles". The Sepsis Bundles are evidence based guidelines that when implemented together, as a group, are expected to improve the outcome of septic patients ${ }^{5}$.

There are two sets of sepsis bundles, sepsis resuscitation bundle which should be implemented within the initial 6 hours after patient admission and sepsis management bundle which should be implemented as soon as possible but within the first 24 hours.

\section{SEPSIS RESUSCITATION BUNDLES}

These are evidence based interventions that must be completed within 6 hours for patients with

a) Severe Sepsis

b) Septic shock and

c) Serum Lactate $>4 \mathrm{mmol} / \mathrm{L}$

The following are the evidenced based interventions that are recommended during the first six hours in a patient with severe sepsis.

\section{Measure Serum Lactate}

Serum lactate measurement is recommended as the first priority in patients with severe sepsis so as to start hemodynamic support if the serum lactate is $>4 \mathrm{mmol} / \mathrm{L}$ regardless of blood pressure $^{6}$. As serum lactate can be measured easily and accurately, it is recommended to use lactate measurement to monitor severity of sepsis and to guide therapy ${ }^{7,9}$.

\section{Blood cultures obtained prior to antibiotic administration}

Blood should be obtained for culture prior to antibiotic administration in critically ill septic patients. This offers the best chance to identify organism that caused severe sepsis in any patient. It is recommended to obtain two or more blood cultures ${ }^{8}$.

\section{Improve time to broad spectrum antibiotics}

Broad spectrum antibiotic should be administered within 3 hours for patients admitted to the emergency department and within one hour when admitted to the ICU setting. This should cover all suspected possibilities of infection and is based on possible or demonstrated focus of infections, likely microorganisms etc. The antibiotic therapy needs to be adjusted within 48-72 hours once the responsible organism and its antibiotic susceptibilities are identified ${ }^{9}$.

\section{Treatment of hypotension}

\section{a) Fluid Resuscitation}

If these patients are hypotensive or have a serum lactate of more than $4 \mathrm{mmol} / \mathrm{L}$ then a initial fluid bolus of $20 \mathrm{ml} / \mathrm{kg}$ is given. These fluid boluses are repeated as necessary until the end points of resuscitation are achieved. These are a MAP of $>65 \mathrm{mmHg}$, heart rate $<110 / \mathrm{min}, \mathrm{CVP} \geq 8$ mmHg. There is no outcome difference when crystalloids or colloids are used for fluid boluses in septic patients ${ }^{10}$. If the hypotension is persisting a central venous line is needed to monitor central venous pressure. Further fluid boluses are given to bring the CVP to $>8 \mathrm{mmHg}^{6}$. If the intrathoracic and intraabdominal pressures are elevated then CVP of $12-15 \mathrm{mmHg}$ is desirable.

It is also recommend to measure central venous oxygen saturation and if it is less than $70 \%$ an attempt should be made to increase it by

1. Packed cells transfusion to bring the Hct to $30 \%$

2. Dobutamine infusion upto $20 \mathrm{ug} / \mathrm{kg} / \mathrm{min}$ to increase cardiac index

3. Decreasing oxygen demand by sedation and mechanical ventilation if red blood cells and inotropic drugs fail to bring the central venous oxygen saturation to more than $70 \%$.

\section{b) Vasopressor therapy}

Vasopressors are usually administered to patients who fail to maintain a MAP $>65 \mathrm{mmHg}$ despite adequate fluid resuscitation. Dopamine or Norepinephrine infusion is usually the drug of first choice for supporting blood pressure in patients with septic shock.

\section{SEPSIS MANAGEMENT BUNDLE}

\section{Low Dose Steroid Administration}

Surviving sepsis campaign recommended the use of stress dose of corticosteroids for septic shock regardless of adrenal function ${ }^{12}$. Patients who require vasopressors to maintain blood pressure despite adequate fluid resuscitation are given intravenous hydrocortisone 200-300 mg/day for 7 days in 3 or 4 divided doses or by continuous 
infusion. Low dose corticosteroid for short or long periods does not significantly increase the risk of gastrointestinal bleeding, hyperglycemia, or super infections ${ }^{13}$.

\section{Activated Protein C Administration (rhAPC)}

rhAPC, an endogenous anticoagulant with anti inflammatory properties showed improved survival in patients with sepsis induced organ dysfunction. The PROWESS ${ }^{14}$ study using rhAPC demonstrated a $15 \%$ reduction in the relative risk of 28 days all cause mortality. rhAPC is recommended in patients at high risk of death (APACHE II $>25$ ), sepsis induced multiple organ failure, septic shock, sepsis induced acute respiratory distress syndrome and no absolute contraindications related to risk of bleeding or relative contraindications that outweighs the potential benefit of rhAPC.

\section{Maintain Adequate Glycemic Control}

Another therapeutic strategy that has been found to confer a mortality benefit to critically ill and predominantly postsurgical cardiothoracic patients is tight glycemic control ${ }^{15}$ It is recommended to maintain blood glucose less than150 $\mathrm{mg} / \mathrm{dL}(8.3 \mathrm{mmol} / \mathrm{L})$ following initial stabilization of patients with severe sepsis. In patients with severe sepsis, a strategy of glycemic control should include a nutrition protocol with the preferential use of the internal route.

\section{Lung protective ventilation strategies}

Experimental data have revealed that large tidal volume ventilation can stretch lung tissue, exacerbating the inflammatory response and leading to acute lung injury. ${ }^{16}$ Since sepsis is the most common cause of ARDS, it is recommended to institute ventilation with low tidal volumes when lung injury or ARDS is present. It is desirable to keep the plateau pressure to less than $30 \mathrm{cms}$ of water in patients with severe sepsis requiring mechanical ventilation ${ }^{17}$ so as to avoid the development of ventilator induced lung injury.

Conclusion Severe sepsis and septic shock are commonly seen in clinical practice .With the advancement in the knowledge and early recognition of these conditions outcome can be improved by instituting sepsis bundles early and appropriately. There is a need to form a team taking care of such patients and treatment should be started as soon as possible after recognition of patients with severe sepsis. Sepsis resuscitation bundle needs to be implemented during the first six hours to optimize outcome. Other therapies that can afford a mortality benefit for patients with severe sepsis and septic shock include the administration of corticosteroids, activated protein $\mathrm{C}$, mechanical ventilation with low tidal volumes, and tight glycemic control

\section{References}

1. Angus DC, Line-Zinirble WT, Lidicker J, Clement G, Carcillo J, Pinsky MR. Epidemiology of severe sepsis in the united states: analysis of incidence, outcome, and associated costs of care. Crit Care Med 2001; 29: 1301-1310.

2. Brone RC, Balk RA, Cerra FB et al. Definitions for sepsis and organ failure and guideline for the use of innovative therapies in sepsis. The ACCP/SCCM Consensus Conference Committee, Chest 1992; 101: 1644-1655.

3. Estenssoro E, Gonzalez F, Laffaire E, Canales H, Saenz G, Renia R et al. Shock on admission day is the best predictor of prolonged mechanical ventilation in the ICU. Chest 2005; 127: 598-603.

4. Brun-Buisson C, Doyon F, Carlet J, Dellamonica P, Gouin F, Lepoutre A et al. French ICU Group for severe sepsis. Incidence risk factors and outcome of severe sepsis and septic shock in adults. A multicentre prospective study in intensive care units. JAMA 1995; 274: 968-974.

5. Levy MM, Provonost PJ, Dellinger RP et al. Change bundles: converting guidelines into meaningful change in behavior and clinical outcome. Crit Care Med 2004: pp S595-S597.

6. Rivers E, Nguyen B, Havstad S et al. Early goal directed therapy in the treatment of severe sepsis and septic shock. New England Journal of Medicine 2001; 345: 1368-1377.

7. Bakker J, Coffernils M, Leon M et al. Blood lactate levels are superior to oxygen derived variables in predicting outcome in human septic shock. Chest 1991; 99: pp 956-962.

8. Weinstein MP, Reller LP, Murphy JR et al. The clinical significance of positive blood cultures: A comprehensive analysis of 500 episodes of bacteremia and fungemia in adults. Laboratory and epidemiologic observations. Rev Infect Dis 1983; 5: 35-53.

9. Bochud PY, Bonten $\mathrm{M}$, Marchetti $\mathrm{O}$ et al. Antimicrobial therapy for patients with severe sepsis and septic shock: an evidence base review. Crit Care Med 2004; 32: pp S495-S512. 
10. Choi PT, Yip G, Quinonez LG et al. Crystalloids vs. Colloids in fluid resuscitation: a systemic review 1999: pp 200-210.

11. Beale RJ, Hollerberg SM, Vincent JL et al. Vasopressor and inotropic support in septic shock: an evidence based review. Crit Care Med 2004: 32; pp S455-S465.

12. Annane D, Sebille V, Charpentier C et al. Effect of treatment with low doses of hydrocortisone and fludrocortisones on mortality in patients with septic shock. JAMA 2002: pp 862-871.

13. Annane D, Bellisant E, Bollaert PE et al. Corticosteroids for severe sepsis and septic shock: a systemic review and Meta analysis. BMJ 2004 329: pp 480.

14. Bernard GR, Vincent JL, Laterre PF, et al. Efficacy and safety of recombinant human activated prokein C for severe sepsis. $N$. Eng $J$ Medicine 2001; 344: pp 699-709.

15. Van den Berghe G, Wouters P, Weekers F, Verwaest C, Bruyninckx F, Schetz M, et al. Intensive insulin therapy in the critically ill patients. N Engl J Med 2001; 345: 1359-67

16. Frank JA, Matthay MA. Science review: mechanisms of ventilator-induced injury. Crit Care 2003;7:233-41

17. The Acute Respiratory Distress Syndrome Network: Ventilation with lower tidal volumes as compared with traditional tidal volumes for acute lung injury and the acute respiratory distress syndrome. $N$ Engl $J \quad$ Med 342.13011308.2000;18 